\title{
Survey of Health Care Context Models and Prototyping of Healthcare Context Framework
}

\author{
Hamid Mcheick \\ University of Quebec at \\ Chicoutimi \\ 555 Boul. University, \\ Chicoutimi (QC) G7H 2B1 \\ Canada \\ Hamid_mcheick@uqac.ca
}

\author{
Hicham Ajami \\ University of Quebec at \\ Chicoutimi \\ 555 Boul. University, \\ Chicoutimi (QC) G7H 2B1 \\ Canada \\ Hicham.ajami1@uqac.ca
}

\author{
Zayan Elkhaled \\ University of Quebec at \\ Chicoutimi \\ 555 Boul. University, \\ Chicoutimi (QC) G7H 2B1 \\ Canada \\ Zayan.elkhaled1@uqac.ca
}

\begin{abstract}
HealthCare system is considered a key element in ubiquitous computing, which means health care services are recommended at any time and at any places to monitor patients based on their context. This study aims to define a simple framework that could be guidelines when you start thinking about building a health context-awareness system in ubiquitous environment. This paper starts by defining the concept of context and then presenting a comprehensive list of context models. Outcome of the work of this research is an ontology healthcare model based on current context of patient which makes monitoring process more accurate. This study includes an Android application in order to prove the concept of this hypothesis.
\end{abstract}

\section{Author keywords}

HealthCare; pervasive computing; Context models; Context representation; Telemedicine; Health care frameworks;

\section{ACM Classification Keywords}

1.6.1 LIFE AND MEDICAL SCIENCES: Health Medical Systems. See: http://www.acm.org/about/class/1998/ for more information and the full list of ACM classifiers and descriptors.

\section{INTRODUCTION}

The current development of the pervasive computing helps to create new generations of services and represents a significant opportunity to advance our society to be more comfortable and safer in different areas of life.
SummerSim-SCSC, 2016 July 24-27, Montreal, Quebec, Canada C 2016 Society for Modeling \& Simulation International (SCS)
One of the most important parts of pervasive computing is that related to healthcare systems.

The healthcare computing transmits the physiological parameters of patients via the modern communication technologies that allow the caregivers to be able to do the necessary procedures in emergency cases. In this paper, mainly we will illustrate the importance of user activity as a key factor of the context components. The proposed system is a mobile application providing the healthcare servers by contextual data gathered by sensors in order to monitor the current status of patients. This article is organized as follow; Section 2 gives a literature review of existing context's definition and its representations models. Section 3 describes healthcare frameworks based on extended ontology and presents a well-detailed case study. In the last section we conclude this work and give some additional perspectives.

\section{LITERATURE REVUE}

\section{Definitions of context}

The context has captured attention of researchers in many fields such as philosophy, sociology, art, history and religion as well as information technology. Apart from all of these meanings that do not help much for understanding our targeted context, this work is only interested to the context related to ubiquitous computing environment. To be aware of the context components is the most important step in going towards building the pertinent models or developing applications in such ubiquitous system. The notion of context has appeared implicitly for the first time in ubiquitous computing area in 1993 by Weiser "all the information that should be taken into consideration for an adjustment". The formal definition of context which is widely accepted was given by Dey et al. [1]: "Context is any information that can be used to characterize the situation of an entity. An entity is a person, place, or object that is considered relevant to the interaction between a user and an application, including the user and applications themselves". In 2015 [13] provided the latest definition of context "is any piece of information that 
can represent changes of the circumstance (either static or dynamic). Also, Chaari et al. 2005 defined the context as a set of external parameters to the application that can influence behavior by defining new views of its data and services [31]. In this paper, we adopt this definition of context.

\section{How to represent the Context: context models}

The context model or context representation refers to the approach that describes and organizes the contextual information to facilitate the work inside the context awareness system. Strang et al. [14] presented six main methods for constructing context models: Key Value Models, Mark-up Scheme Models, Graphical Models, Object Oriented Models, Logic Based Models, and Ontology Based Models. Bettini et al. [15] investigated three different approaches to depict the context: Object-role, Spatial and Ontology. Khattak et al. [16] argued that most of literature surveys on context representation modeling are incomplete where authors omitted the Spatial Representation, Hybrid and Domain Focused models. Hence, they proposed nine different methods to represent encountered information. Later in 2015, Li et al. added 3 new models called user centric, Chemistry inspired, and Multidisciplinary. The recent surveys related to context models demonstrate the existence of six to twelve models, which are given in table 1 .

\begin{tabular}{cccc}
$\begin{array}{c}\text { Strang } \text { et al. } \\
\mathbf{2 0 0 4}\end{array}$ & $\begin{array}{c}\text { Bettini } \text { et } \\
\text { al. 2009 }\end{array}$ & $\begin{array}{c}\text { Khattak } \text { et al. } \\
\mathbf{2 0 1 4}\end{array}$ & $\begin{array}{c}\text { Li } \text { et al. } \\
\mathbf{2 0 1 5}\end{array}$ \\
\hline $\begin{array}{c}\text { Key-Value } \\
\text { Mark-up }\end{array}$ & $\begin{array}{c}\text { Object-role } \\
\text { Spatial }\end{array}$ & $\begin{array}{c}\text { Key-Value } \\
\text { Mark-up }\end{array}$ & $\begin{array}{c}\text { Key-value } \\
\text { Mark-up Scheme }\end{array}$ \\
Graphical & Ontology & Graphical & Graphical \\
Object Oriented & Hybrid & Object Oriented & Object Oriented \\
Logic-Based & & Logic Based & Logic Based \\
Ontology & & Ontology & Ontology \\
& & Spatial & Domain focused \\
& & Domain-focused & User Centric \\
& & Hybrid & Multidisciplinary
\end{tabular}

Table 1: Context models.

\section{Key-value model}

It is one of the easiest ways of modeling the context because it is based on the principle of mapping that is matching the contextual information with a numerical value or attributes. "Key-value coding is a mechanism for accessing an object's properties indirectly, using strings to identify properties, rather than through invocation of an access method or accessing them directly through instance variables" [21]. Mark-up Scheme Model
It is known as tagged encoding where context information is stored within tags issued by typical mark-up languages such as XML. Mark-up Scheme is useful for modeling profiles for example (device capabilities). Usually, those attributes and tags can be interpreted according to their position in the data tree; due to the hierarchical structure of this method [22].

\section{Logical model}

This model depicts the contextual data or properties as fact, terms, rules or expressions derived from first or second order logic to set controls policies that conclude the reasoning context. Logic-based is a specific class of contextual information models where logic defines the status that may be derived from a set of other expressions or facts [23].

\section{Object Oriented Representation Model}

The cornerstone of this model is the object-oriented programming paradigm that represents data structures as objects (classes) with different attributes, "The basic idea behind OOP is the combination of data and the functions that operate on that data into one single entity" [24].

\section{Graphical model}

This model represents the context as diagrammatical scheme or in graphical notation; the common graphical models used are the Unified Role Modeling (UML), Entity Relationship Model (ERM) and Object Role Modeling (ORM) [14]. This scheme includes diagrammatical representation of context at the design time that should be translated into a usable format [16].

\section{Ontology}

The term ontology means existence of things, but in the pervasive computing, [25] defined Ontology as follows: "ontology is a formal, explicit specification of a shared conceptualization". Actually Ontology could be represented by two description languages: RDF (Resource Description Framework) and OWL (Ontology Web Language)Usually, the ontology is divided into a two-level hierarchy; the high level describes general concepts of the ontology while the lower level is re-divided into several pervasive computing sub-domains e.g. home domain, transport domain [18].

\section{Domain Focused}

This model is widely known as W4, where this approach proposed a general context representation for expressing facts by the main 4-fields which is answering the following question: Who was the user, what was the activity being performed, Where the activity was performed (location), When the activity was performed (time).

\section{Spatial Representation}

Spatial or location is one of the most typical contextual information. Often context-aware application uses location of people, objects, or devices as a main source of their targeted contextual information [17].

Typically, there are two kinds of location coordinate which 
are supported by positioning systems Geometric and symbolic coordinates [15].

\section{Multidisciplinary model}

Li et al. [13] defined the multidisciplinary as demonstration of context from different points of view, identifying the relationships among multiple disciplines. [19] Argued that multidisciplinary approach is the best way to address the emanating issues of the transition from traditional static desktop computing to heterogeneous mobile environments. This method is tailored for applications in which human and social issues should be identified.

\section{User-centric}

This model is based on user's perspective instead of computing devices, or even applications. In this context the authors of [13] described user centric as 5W1H-tuple: Who, When, Where, What, How, Why. This method is quite useful to discover a meaningful context by inferring user's intention based on combination of integrated context [27]. This design Suits applications focused on perspectives of users; data expression is in an intuitive manner.

\section{Chemistry inspired Model}

It is called chemistry inspired because it is similar to chemical reaction, where it represents its context and triggers the right services dynamically. In 2011 Fernandez [30] said "Chemistry-inspired computing recently regained momentum in this context, naturally expressing parallelism, distribution, and autonomic behaviors". Later [28] presented the chemistry inspired as a parallel interaction model or a form of collective intelligence between system components to enable them to achieve a common objective.

\section{Hybrid model}

The hybrid model is an integration of two or more existing context representation models in order to obtain flexible and general models. Paganeli et al. [29] proposed a hybrid technique which aims to benefit from the advantages of the object-oriented models and ontology-based models. [15] attempted to combine different formalisms and approaches to get the identified requirements by introducing architecture from multi-layer framework allow using mark-up scheme in layer2 with ontology in the layer 3 .

\section{Comparison of context model features}

The tables 2, 3 and 4 are an evaluation of context models presented by Bettini et al., Strang et al. and Xin Li et al. respectively.

$\begin{array}{llll}\text { Models } & \text { Object-role } & \text { Spatial } & \text { Ontological } \\ \text { Mobility } & \begin{array}{l}\text { Partial } \\ \text { satisfaction }\end{array} & \begin{array}{l}\text { Satisfied } \\ \text { satisfaction }\end{array} & \text { Unsatisfied } \\ \text { Relationships } & \begin{array}{l}\text { Partial } \\ \text { satisfaction }\end{array} & \begin{array}{l}\text { Partial } \\ \text { satisfaction }\end{array} & \text { Satisfied } \\ \text { Timeliness } & \text { Satisfied } & \text { Satisfied } & \text { Unsatisfied } \\ \text { Imperfection } & \begin{array}{l}\text { Partial } \\ \text { satisfaction }\end{array} & \begin{array}{l}\text { Partial } \\ \text { satisfaction }\end{array} & \text { Unsatisfied } \\ \text { Reasoning } & \begin{array}{l}\text { Partial } \\ \text { satisfaction }\end{array} & \text { Unsatisfied } & \text { Satisfied } \\ \text { Usability } & \text { Satisfied } & \text { Partial } & \text { Partial } \\ & \text { Patisfaction } & \text { satisfaction } \\ \text { Efficiency } & \text { Partial } & \text { Satisfied } & \text { Unsatisfied } \\ & \text { satisfaction } & & \end{array}$

Table 2: A comparison of context modeling approaches [15].

\begin{tabular}{|c|c|c|c|c|c|c|}
\hline Criteria & dc & pv & qua & inc & for & app \\
\hline Key-value & - & - & - & - & - & + \\
\hline Markup & + & ++ & - & - & + & ++ \\
\hline Graphical & - & - & + & - & + & + \\
\hline $\begin{array}{c}\text { Object } \\
\text { Oriented }\end{array}$ & ++ & + & + & + & + & + \\
\hline Logic based & ++ & - & - & - & ++ & - \\
\hline Ontology & ++ & ++ & + & + & ++ & + \\
\hline
\end{tabular}

Table 3: Appropriateness Indication [14].

Ubiquitous computing systems make high demands on any context modeling approach in terms of

1. distributed composition (dc)

2. partial validation ( $\mathrm{pv})$

3. richness and quality of information (qua)

4. incompleteness and ambiguity (inc)

5. level of formality (for)

6. applicability to existing environments(app)

In the next table, we used "+" to denote the kind of characteristic to be supported, "-" to denote the kind of characteristic not to be supported, and "o" to denote the kind of characteristic when it is not clear whether or not it should be supported.

\begin{tabular}{llllllll} 
Criteria & Ex & Rs & Rt & In & Fm & Va \\
\hline
\end{tabular}




$\begin{array}{lcccccc}\text { Key-Value } & - & - & - & 0 & - & 0 \\ \text { Mark-up Scheme } & - & - & + & 0 & + & + \\ \text { Graphical } & + & 0 & + & - & - & 0 \\ \text { Object Oriented } & - & - & + & 0 & - & - \\ \text { Logic } & + & + & 0 & 0 & - & - \\ \text { Multidisciplinary } & + & \mathrm{o} & \mathrm{o} & - & \mathrm{o} & - \\ \begin{array}{l}\text { Domain focused } \\ \text { User Centric }\end{array} & + & \mathrm{o} & \mathrm{o} & \mathrm{o} & - & - \\ \text { Ontology } & + & + & \mathrm{o} & \mathrm{o} & - & - \\ \text { Chemistry } & + & + & + & + & + & + \\ & + & \mathrm{o} & \mathrm{o} & \mathrm{o} & - & -\end{array}$

Table 4: A comparison of context modeling approaches [13].

\section{E: Expressiveness \\ 2. Rs: Reasoning \\ 3. Rt: Relationships \\ 4. In: Interoperability \\ 5. Fm: Formalism \\ 6. Va: Validation tools}

\section{Analysis of these models}

The bottom line and based on the foregoing, the effective representation is the one that fulfill the maximum requirements to simulate realistic picture of the context. Some models represent context in simple manner but sometimes fail to explain the complex data, such as key value models which considered useful for simple standalone applications[16] or mark-up that is only efficient with low level of contextual information[14]. Turning to logic model we can easily find out that although of its richness of expression, it suffers from some problems such lack of standardization and validation tools. As for object oriented model is suitable for a dedicated application with no reasoning feature. The graphical model plays an important role within database managements that holds a massive amount of data, through executing quick database queries, but besides these pros, this technique is a flat information model, does not support distribution and it limits the interoperability and privacy. The recent representation of context is moving toward ontology due to its efficiency with data complexity and heterogeneity of sources, moreover, ontology uses descriptive logic to represent that knowledge which is easy to handle. But, the ontology as well lacks of fuzziness and incapable for handling of uncertainty [16]. The domain-focused provides an easy representation structure with simple processing while the cons of such strategies varies from flexibility weakness and system adaptability, where it is only appropriate to single domain applications. Spatial or location able to cope with the context complexity and equipped with reasoning process, the main drawback of spatial model is the painstaking effort to collect the location data and to keep it up to date. The last three models mentioned by [13] Multidisciplinary, User-centric, and Chemistry inspired models still at the first stage and has no validation proof in addition to being difficult to use. As we discussed above in this section, each model has advantages and disadvantages. Depending of following requirements, we have to choose the context model that satisfies the most of them [14] distributed composition, the partial validation, the richness and quality of information, the incompleteness and ambiguity, level of formality and applicability of the existing environment. In contrast, [15] argued different range of characteristics such as heterogeneity, usability timeless; relationship and reasoning that should be available in the ideal representation depend on the priority of your model. In general, the hybrid approaches often work well where they combine the benefits and important characteristics of the mentioned models. But [16] thinks that even hybrid model is suffering from two main problems the first problem is the representation of flawed context information and the second problem is lack of privacy where preferences are exposed.

\section{Context aware HealthCare frameworks}

Bricon-Souf et al. [2] considered the context-awareness computing is a research field which often refers to health care as an interesting and rich area of application. The recent works that have been done before were valuable and provided significant achievements in this domain. Abidoy et al. [3] presented a complete architecture of wearable sensors for remote healthcare monitoring system composed of three tiers based on physiological signal characteristics such as (Electrocardiograph, Blood Flow Respiratory Rate, breaths/min, Oxygen Saturation (SpO2), Blood Pressure, Blood PH Never Potentials, Body Temperature) and provide real-time feedback to the user and the medical staff. Kang et al. [4] proposed a wearable context aware framework as a middleware for ubiquitous healthcare service based on ontology context model for semantic interoperability between sensors and services using the OSGi platform. Another research has been made by [5] which focused on the sensitivity of context aware in the healthcare sector, and it proposed a hybrid model composed of XML and object model for static and dynamic elements of context. For instance, the patient's physiological signals are very sensitive, so any leakage of data could make the report or the evaluation inaccurate and unreliable. The main goal of this project is extending the context's elements in order to build an integrated distributed system free of defects as much as possible. Is this doable? The assumed Answer is coming in the next paragraphs.

\section{System Architecture}

The proposed system is a platform for remote monitoring of wearable sensors, which consists of implantable devices equipped with wireless telemetry. The system allows patients to send comprehensive sensors data to the healthcare centers from their home or wherever they are. The organization of the platform and how patients and health care professionals interact are shown in Figure 1. 


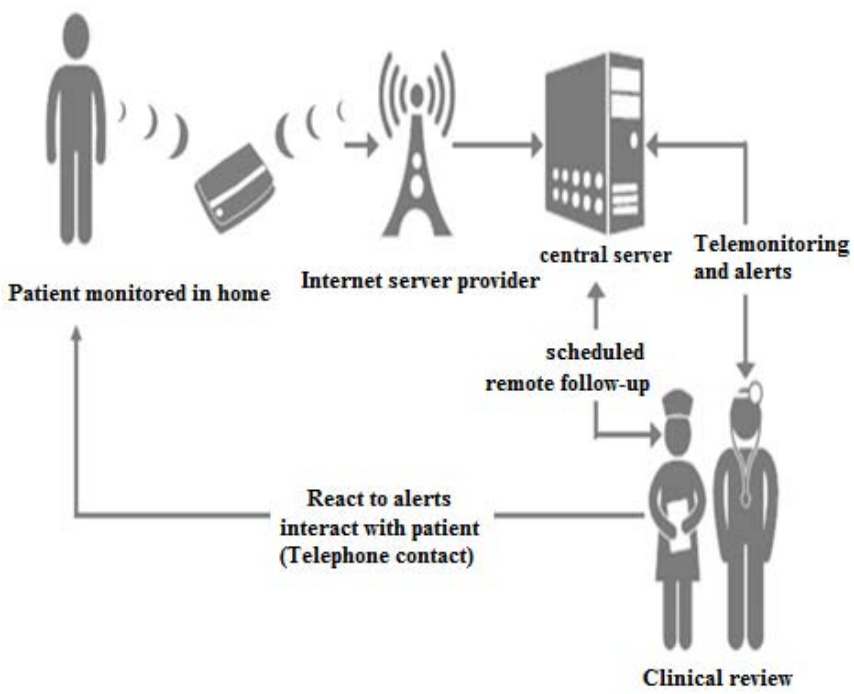

Figure1: Remote monitoring systems [6].

Graschew et al. [7] enumerated a set of the critical wireless sensors standard such as ZigBee, Medical Implant Communications Service (MICS), and Wireless Medical Telemetry Service (WMTS) etc. The Personal Digital Assistant has the capability to hold patient physiological vital signal to the IP medical server through long range communications protocols provided by the existing mobile telecommunications technology such 4G,3G, GPRS, and WAN. On the other hand, the emergency cases are detected by analyzing the data in the healthcare system, which is responsible of sending warning messages to the emergency services centre in order to provide direct assistance to the patient [8] notably from some previous projects such as [20] that overlooked the user activity from their medical context, while others research for example [21] has added the activity as additional features to monitor the current situation of patient without explaining its real effect on the other physiological factors though this element has a very important impact, which is confirmed by medical sources itself.

\section{Context model and case study}

Typically the basic remote healthcare applications aim to control of temperature and heart rate in order to be alert to any unstable state such as an abnormally high or low body temperature and increase or decrease heart rate. As it known to all, the normal body temperature range is $97^{\circ} \mathrm{F}$ to $99^{\circ} \mathrm{F}$ $\left(36.1^{\circ} \mathrm{C}\right.$ to $\left.37.2^{\circ} \mathrm{C}\right)$, the average temperature being $98.6^{\circ} \mathrm{F}$ $\left(37^{\circ} \mathrm{C}\right)[9]$.

\begin{tabular}{ll} 
Age & Average Temperature \\
\hline Adult & $98.6^{\circ} \mathrm{F}-37^{\circ} \mathrm{C}$ \\
Elderly (over $70 \mathrm{yr})$ & $96.8^{\circ} \mathrm{F}-36^{\circ} \mathrm{C}$
\end{tabular}

Table 5: Normal body temperature.

While the normal average heart rate at rest is:

1. Children (ages $6-15$ ) $70-100$ beats per minute
2. Adults (age 18 and over) $60-100$ beats per minute But there are other important factors like physical activity, and location, time of the day and breathing rate could greatly affect the body temperature and heart rate even of healthy persons. In this part we will try to determine the positive or negative correlation between the activity on the temperature and heart pulse. The table below presents the maximum heart rate during intense activities by age [10].

\begin{tabular}{lll} 
Age & Target HR Zone & Maximum Heart Rat \\
\hline 20 years & $100-170$ beats per minute & 200 beats per minute \\
30 years & $95-162$ beats per minute & 190 beats per minute \\
35 years & $93-157$ beats per minute & 185 beats per minute \\
40 years & $90-153$ beats per minute & 180 beats per minute \\
45 years & $88-149$ beats per minute & 175 beats per minute \\
50 years & $85-145$ beats per minute & 170 beats per minute \\
55 years & $83-140$ beats per minute & 165 beats per minute \\
60 years & $80-136$ beats per minute & 160 beats per minute \\
65 years & $78-132$ beats per minute & 155 beats per minute \\
70 years & $75-128$ beats per minute & 150 beats per minute
\end{tabular}

Table 6: Heart rate during hard physical activity.

On the other hand, during the physical exercise, the core temperature can rise to as high as 40 degrees Celsius or 104 degrees Fahrenheit [11].

\section{Modeling Health care}

The ubiquitous healthcare computing systems require context models to ensure the distribution, heterogeneity, the richness and quality of information, ambiguity and applicability of the existing environment.

Arguably that most studies indicate to the superiority of ontology over the other models where the requirements listed above can be achieved, but in spite of all these advantages, Bettini et al. [15] assure that "ontological models alone are generally unsuited to the recognition of basic physical activities; In order to recognize such context data, ontological models should be integrated with statistical machine learning methods".

In this work we adopt and extend a simple ontology-based model where our system monitors the movement of patient as a hard physical activity more than activity recognition. 


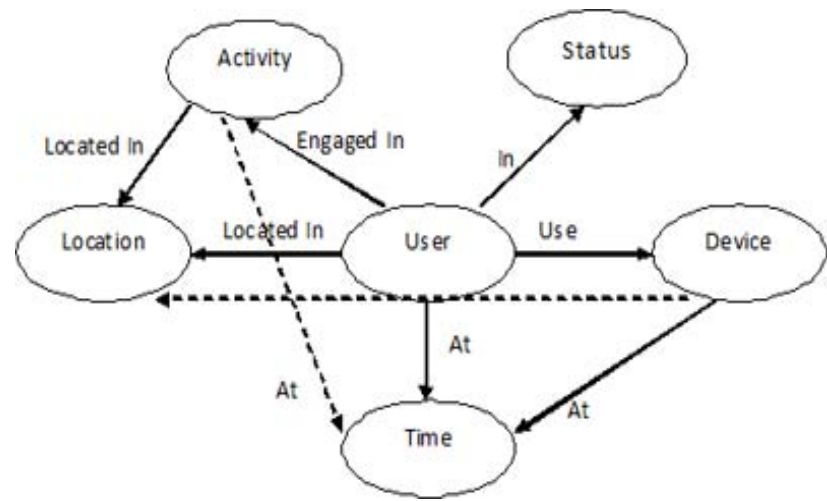

Figure 2: Ontology of Healthcare.

The status is used to describe the health status of user including heart rate, temperature, respiration and blood pressure etc. that could be detected by the connected sensors, while the user specifications can be identified by his own profile (name, age), and the activity can be recognized by acceleration sensors. This is followed by the reasoning process at the server side that translates the representation of context into useful information to support the goal context through inference engine, which is based on specific rules and conditions.

\section{CASE STUDY AND DISCUSSION}

To prove the feasibility of the proposed model, a simple application is provided which is applied to a remote monitoring serve and self-check service mobile.

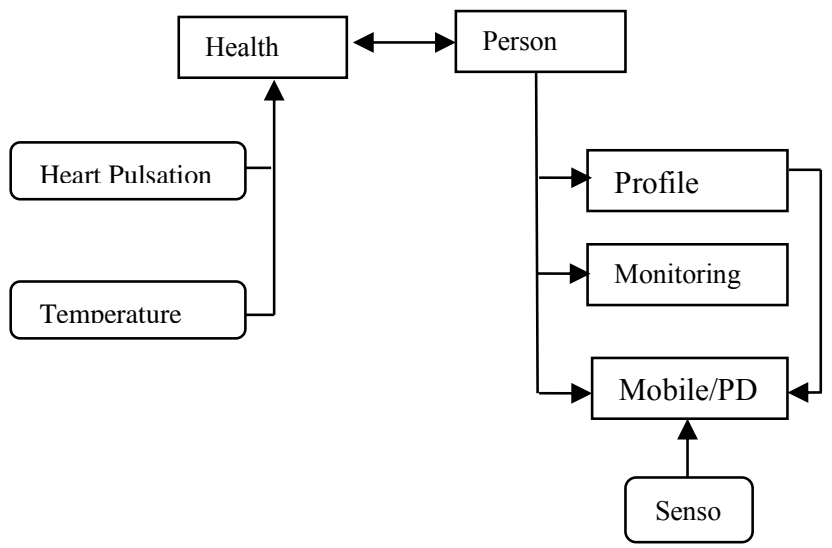

Figure 3: Diagram of application.

The application that has been developed is dedicated for context sensing, especially for activity monitoring of a patient. This distributed system consists of multiple sensors such as temperature, heart monitoring, accelerometer, etc. that should be attached to the human body.

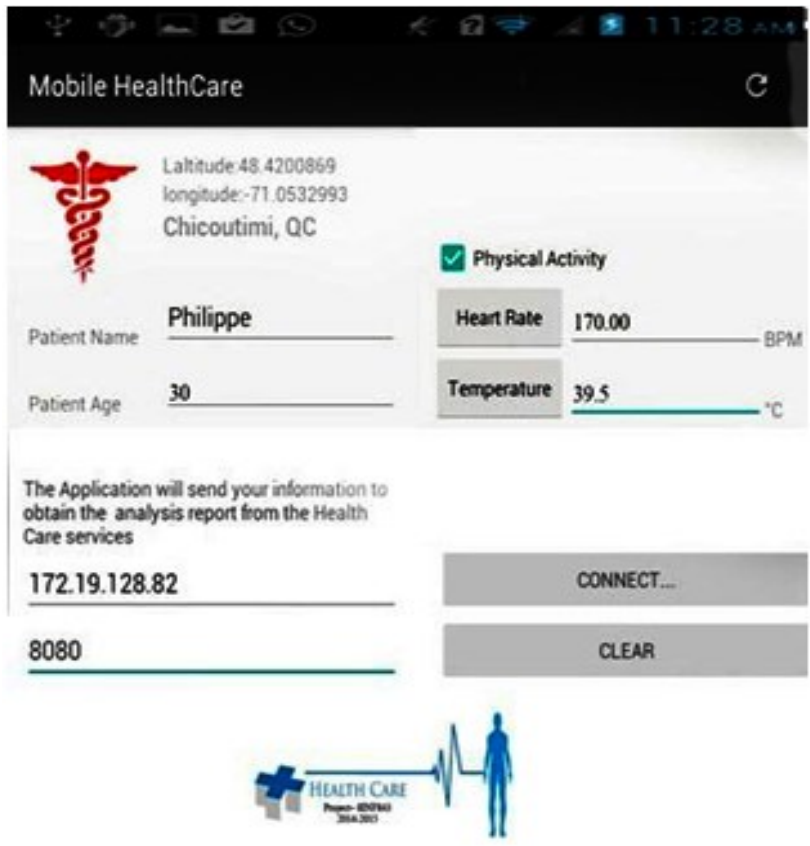

Figure 4: Patient application.

The mobile sensors receive the contexts of heart rate, body temperature, and the current location of patient, in addition to the actual movement. At the server side we developed a monitoring interface, where user's applications connect the available servers through their IP address, to let doctors or nurses to follow the patient status using the real time contexts information transmitted. These data are illustrated by two line graphs based on a set of specific rules (fig.5-a). The patient can check his current status through the same application that provides a detailed diagnosis as we can see in the figure.5-b.
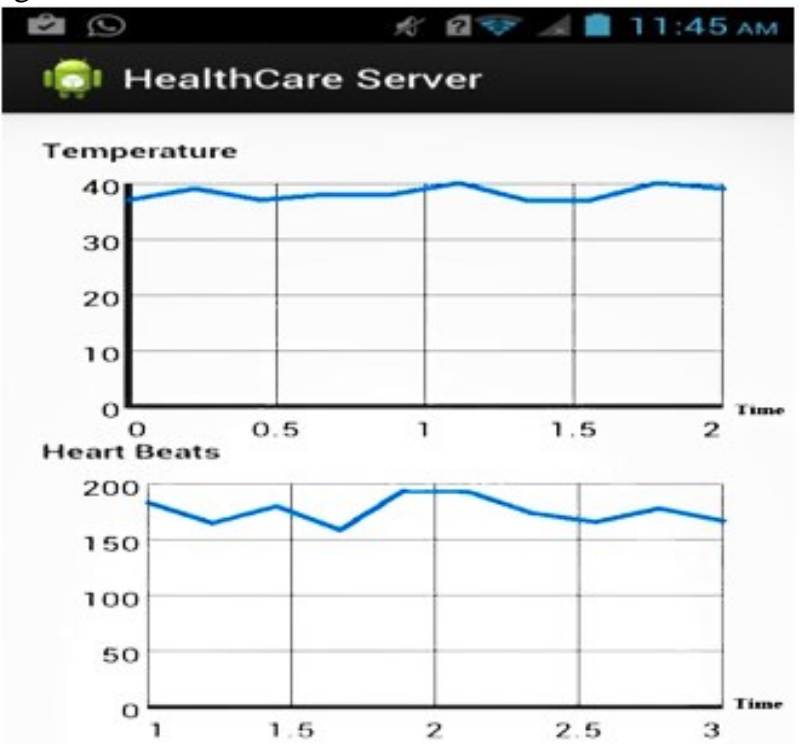

Figure 5-(a): Real time monitoring on server side. 


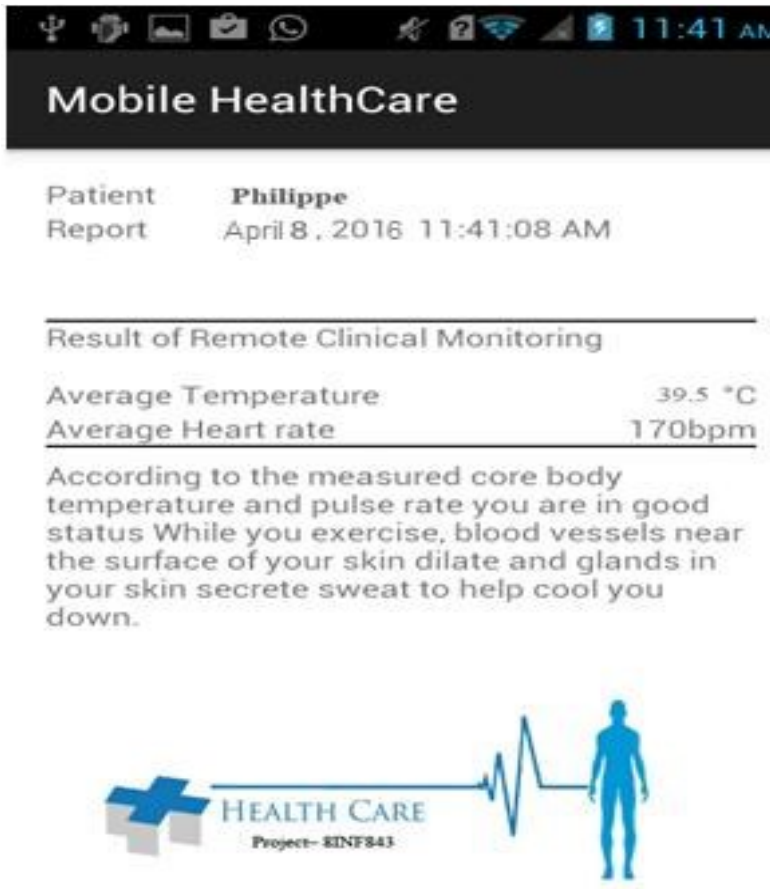

Figure 5-(b): Diagnosis on the client side.

In the conventional system that is based only on physical factors the server or the monitoring entity may generate a false alarm because it assumes there is a risk threatens the health of patient. But in fact that is not true because as we have already seen during activity body temperature rises dramatically. The figure above shows the medical analysis received from the server which confirms that the patient is in good status, unlike the traditional application.

\section{CONCLUSION}

This work could be a simple survey of the available context models where we have discussed the basic traditional and modern strategies to express context. The main objective of the paper is proving the importance of the user activity context for the medical applications therefore this article demonstrates the actual value of identified elements of context. But our current report of context' models does not pretend to be completed, the proposed model needs an extra effort to be more efficient and effective to be applied on different types of patients suffering from certain diseases such as ischemic attack and chronic obstructive pulmonary disease.

\section{ACKNOWLEDGMENTS}

This work is supported by the Department of Computer Science and Mathematics at the University of Quebec at Chicoutimi, Quebec Canada.

\section{REFERENCES}

1. Dey, A., Abowd, G., and Salber D., A conceptual framework and a toolkit for supporting the rapid prototyping of context-aware applications. Journal of Human-Computer Interaction, Volume 16 Issue 2 (2001), 97-166.

2. Nathalie Bricon-Souf, Conrad R. Newman. Context awareness in health care: A review. Centre d'Etude et de Recherche en Informatique Medicale, Universite' de Lille. Elsevier Ireland, 2006.

3. Ademola Philip Abidoy, Nureni Ayofe Azeez, Ademola Olusola Adesina, Kehinde K. Agbele, Henry O. Nyongesa." Using Wearable Sensors for Remote Healthcare Monitoring System". Journal of Sensor Technology, 1 (2011), 22-28.

4. Dong-Oh Kang, Kyuchang Kang, Hyung-Jik Lee, EunJung Ko, Jeunwoo Lee. A Context Aware Framework for u-Healthcare in a Wearable System, Post-PC Research Group, Electronics and Telecommunications Research Institute, Daejeon, Korea. Springer, Volume 14 of the series IFMBE Proceedings (2007), pp 40264029.

5. Hamid Mcheick. Modeling Context Aware Features for Pervasive Computing, The 5th International Conference on Emerging Ubiquitous Systems and Pervasive Networks (EUSPN-2014), Journal title: Procedia Computer Science, Procedia Computer Science (2014), pp. 135-142. Elsevier, September 22-25, Halifax, Canada, 2014.

6. Giuseppe Boriani1, MD, PhD; Antoine Da Costa2. The MOnitoring Resynchronization dEvices and CARdiac patiEnts (MORE-CARE) Randomized Controlled Trial: Phase 1 Results on Dynamics of Early Intervention With Remote Monitoring. JMIR Publications, Published on 21.08.2013 in Vol 15, No 8 (2013).

7. Georgi Graschew and Stefan Rakowsky. Telemedecine techniques and applicationsS. Published by. InTech, Chapters, published June 20, 2011 under CC BY-NCSA 3.0 license.

8. Shyamal Patel, Hyung Park,3 Paolo Bonato, Leighton Chan, and Mary Rodgerscorresponding author. A review of wearable sensors and systems with application in rehabilitation. U.S. National Library of Medicine, $J$ Neuroeng Rehabil. V9: 21 (2012).

9. Kathy Bonewit-West, Today's Medical Assistant: Clinical \& Administrative Procedures, 2nd Edition. Vital Signs Elsevier, 2012.

10. American heart association: http://www.heart.org/ last accessed, November 2015.

11. Lee VJ1, Wong CS, Tambyah PA, Cutter J, Chen MI, Goh KT. Twentieth century influenza pandemics in Singapore. Annual Academie Med Singapore. 2008 Jun; 37(6): 470-6. 
12. Schilit, B., Theimer, M. Disseminating Active Map Information to Mobile Hosts. IEEE Network, 8(5) (1994).

13. Xin Li, Martina Eckert, José-Fernán Martinez and Gregorio Rubio. Context Aware Middleware Architectures: Survey and Challenges, Journal Sensors, 2015.

14. Strang, T. and Linnhoff-Popien C. (2004) A Context Modelling Survey. Paper presented at the UbiComp Workshop - First International Workshop on Advanced Context Modelling, Reasoning and Management, 2004.

15. Bettini, C., Brdiczka, O., Henricksen, K., Indulska, J., Nicklas, D., Ranganathan, A., Riboni, D. A survey of context modelling and reasoning techniques. Pervasive Mob. Comput. 6(2), 161-180 (Apr 2010)

16. Asad Masood Khattak, Noman Akbar, Mohammad Aazam, Taqdir Ali,Adil Mehmood Khan, Seokhee Jeon, Myunggwon Hwang and Sungyoung Lee, Context Representation and Fusion: Advancements and Opportunities, Journal of sensors 25 January 2014, 14(6), 9628-9668.

17. Christian hoareau, A model checking based framework for building correct context aware systems, PHD, SOKENDAI University, 2009.

18. Roland Reichle, Michael Wagner, Mohamma, A Comprehensive Context Modeling Framework for Pervasive Computing Systems, Conference: Distributed Applications and Interoperable Systems, 8th IFIP WG 6.1 International Conference, DAIS 2008, Oslo, Norway, June 4-6, 2008.

19. Nicholas A. Bradley and Mark D. Dunlop, Towards a Multidisciplinary Model of Context to Support ContextAware Computing, 2005, Human-Computer Interaction, 2005, Volume 20, pp. 403-446.

20. S. Sridevi, Bhattacharya Sayantani, K. Pal Amutha, Context Aware Health Monitoring System, springer, 2010.

21. A. Wood, J. Stankovic, G. Virone, L. Selavo, Z. He, Q. Cao, T. Doan, Y. Wu, L. Fang, R. Stoleru, ContextAware Wireless Sensor Networks for Assisted-Living and Residential Monitoring, University of Vierginia, 2008 .

22. Sielis, A. G., Mettouris, C., Tzanavari, A. idSpace D3.3 - Definition and implementation of context awareness, v2. 5. LN: Deliverables, Reports, Work Documents, Jan.2009.

23. Max Muhlhauser \& Iryna Gurevych (Author), Max Muhlhauser, Iryna Gurevych. Handbook of Research on Ubiquitous Computing Technology for Real Time Enterprises. Publisher: Information Science Reference; 1 edition, January 17, (2008).
24. George A. Tsihrintzis, Virvou, Lakhmi C. Jain, and Robert J. Howlett. Intelligent Interactive Multimedia Systems and Services, Springer, Vol. 11. 2010.

25. Berufsakademie Mannheim, Information Technology International, Lecture Notes Programming II Part 4 Object Oriented Programming, November 2001February 2002.

26. R. Studer, R. Benjamins, and D. Fensel. Knowledge engineering: Principles and methods. Data \& Knowledge Engineering, 25(1-2):161-198, 1998.

27. Yoosoo Oh, Woontack Woo, User Centric integration of context for a unified context-aware application model. Samsung Electronics Co., Ltd., in S.Korea, Joint sOc-EUSAI conference 2005.

28. Mahmoud ElGammal, Chemistry-inspired, ContextAware, and Autonomic Management System for Networked Objects, The Seventh International Conference on Future Computational Technologies and Applications, 2015.

29. F. paganelli and D.giuli, Context aware information services to suppot tourist communities, Electronics and Telecommunications Department, University of Florence, Florence, Italy 2006.

30. Hector Fernandez; IRISA, Univ. of Rennes 1, Rennes, France, A Chemistry-Inspired Workflow Management System for Scientific Applications in Clouds, IEEE, EScience (e-Science), 2011.

31. Chaari, T., Laforest, F. and Flory A. Adaptation des applications au contexte en utilisant les services web. In Proceedings of the 2th French Speaking Conference on Mobility and Ubiquity Computing, pp.111-118. 2005. ACM. 